\title{
Towards QoE-aware adaptive video streaming
}

\author{
Alisa Devlic*, Pavan Kamaraju ${ }^{\dagger}$, Pietro Lungaro*, Zary Segall*, and Konrad Tollmar* \\ *Mobile Service Lab, Royal Institute of Technology (KTH), Kista, Sweden \\ ${ }^{\dagger}$ Department of Computer Science and Electrical Engineering, University of Maryland, Baltimore County, USA \\ Email: devlic@kth.se,pavan4@umbc.edu, pietro@kth.se, segall@kth.se, konrad@kth.se
}

\begin{abstract}
This paper describes a novel QoE-aware adaptive video streaming method that enhances the viewing experience on mobile devices and reduces cellular network bandwidth consumed by Dynamic Adaptive Streaming over HTTP (DASH) by considering perceptual video quality and data rate channel conditions in the bitrate adaptation process. By streaming an optimized video for the particular video quality and channel conditions to a mobile device, we can improve the worst video qualities caused by DASH streaming and reduce quality variations using fewer number of bits.
\end{abstract}

Keywords-QoE, Adaptive video streaming, DASH

\section{INTRODUCTION}

Deteriorations of video quality experienced during Dynamic Adaptive Streaming over HTTP (DASH) over a varying mobile network channel can degrade a viewers' QoE. The proposed method proactively optimizes a video stream for viewing on a mobile device in the highest perceptual video quality that can be achieved in the given video and channel conditions. The optimized video is streamed to a user's device in constant perceptual video quality, using the remaining throughput to prefetch future seconds of video stream in order to account for potential poor channel performances. In case a segment cannot be downloaded until the end of next second, due to lack of bandwidth or bits in the buffer, QoE-aware streaming switches to DASH at lower qualities. Hence, this should not occur so frequently since an optimized video quality is chosen to reflect statistical properties of the available bandwidth.

By limiting maximum bitrates of requested video segments and prefetching optimized video in advance of playout, this method improves the worst video qualities introduced by DASH and reduces amplitude of video quality variations (as shown in Figure 1), while saving network bandwidth. As users are more sensitive to video quality degradations than they appreciate quality improvements [1] [2], quality of streaming video will be better perceived by increasing the lowest video quality than by increasing any other higher quality level [2]. Observe that the latter is a consequence of DASH's adaptation strategy - maximizing a segment's bitrate to fit the available throughput (see Figure 1 to the right of the intersection).

Since there is no standardized method to measure QoE of delivered video segments that are in aggregate longer than 15 seconds, in order to compare QoE performances of QoE-aware streaming and DASH on 4 minute long video streams, we examined the lower half of distribution of all 15 seconds parts' video quality scores, representing the worst video qualities of both schemes. The assumption that we make is if DASH has worse perceptual video quality than QoE-aware streaming for $50 \%$ of its lowest quality video segments, corresponding to half of the video duration, then the QoE-aware scheme has higher QoE than DASH. This represents the pessimistic scenario, since the exact percentile of the worst video quality for which one scheme is considered to be superior than another remains yet to be determined. Therefore, we show with percentiles all 978-1-4673-7113-1/15/\$31.00 (c)2015 IEEE possible improvements of the lowest video qualities that can be achieved with QoE-aware streaming.

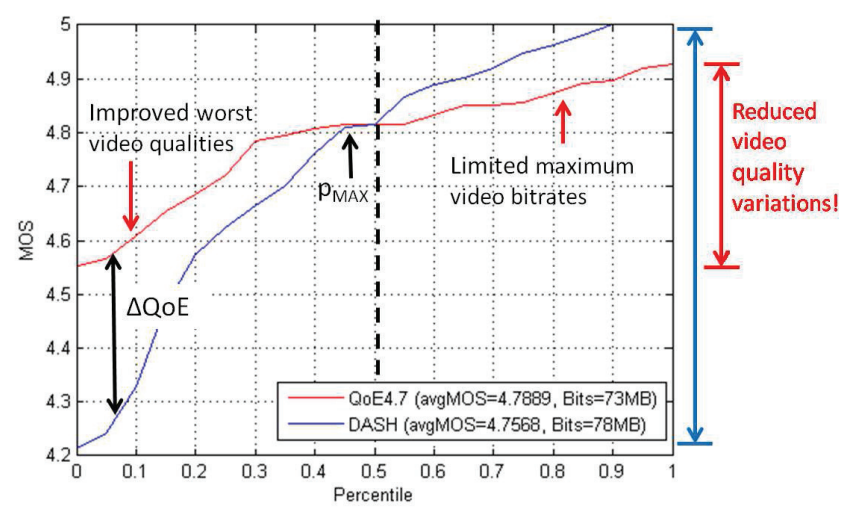

Fig. 1: QoE-aware streaming reduces video quality variations compared to DASH, source: Avengers video, channel 1

$\triangle Q o E$ represents a difference in Mean Opinion Scores $(\mathrm{MOS})^{1}$ of QoE-aware streaming and DASH evaluated in a given percentile of MOS CDF, while the largest percentile for which this difference is equal to or greater than zero (representing the percentage of worst video qualities for which QoE-aware streaming outperforms DASH) is referred to as $p_{M A X}$. Bandwidth savings are computed as a percentage of DASH stream size that is reduced by QoE-aware streaming.

\section{QOE-AWARE STREAMING ALGORITHM}

The QoE-aware streaming requires a video to be optimized in the target perceptual video quality before streaming it to a user's device. The video optimization composes a video of short segments, whose resolution is downscaled from the maximum device supported to the resolution determined by desired perceptual video quality, thus reducing the video size without compromising a viewer's QoE. Due to the limited space, we refer to video optimization in [3] that contains thorough descriptions and investigations of this method.

The existing DASH algorithm from [4] was used as a baseline for implementation of our QoE-aware streaming. This algorithm determines the bitrate for the video segment that should be downloaded next based on estimated bandwidth and occupancy of the playout buffer, using the simple thresholdbased scheme: if the buffer is lower than the predefined threshold $b_{f}$, it selects the highest bitrate that is lower than the current estimated bandwidth. Otherwise, it selects the lowest bitrate that is higher than the current bandwidth.

${ }^{1}$ MOS provides a numerical measure of the perceptual video quality that is usually obtained using subjective video quality tests. The values in Figure 1 were obtained by mapping objective video quality scores of delivered video segments to MOS values using the QoE model from [3]. 
The proposed algorithm starts downloading first video segment in the target video quality, in order to improve the perceived QoE in case of experiencing good channel conditions. $b_{f}$ was set to $1.5^{*} b_{i}$, where $b_{i}$ represents the initial playout buffer size of $2 \mathrm{~s}$ that needs to reached before playback starts.

Our proposed algorithm requires additional inputs:

- $\quad$ critical buffer size, $b_{c}$ - the size of buffer which when reached (or exceeded) signals the algorithm to download the segment in the optimal target video quality, $M O S^{\circ}$, independently of the estimated bandwidth

- $\quad$ average data rate of access channel $^{2}$ for duration of video playback, $\bar{R}$

- bitrates of video segments that are optimized for different target video qualities, $B$

The last two inputs are used to predict the optimal target video quality, $M O S^{\circ}$, for which a video stream should be optimized in order to result in the highest achievable QoE for the given video and data rate channel. This quality is selected as the highest video quality, for which a difference between $\bar{R}$ and optimized segment's bitrate in each second (including a difference from previous seconds which is added to this amount) is positive for at least $90 \%$ of the time, representing the budget of buffered bits available during streaming. The margin of less than $10 \%$ of negative budget values is introduced because of using average instead of actual data rates.

After optimizing a video for the obtained MOS, an MPD file is created with a list of representations specifying video segments in the target and lower qualities, which is upon HTTP request downloaded by the user and used to stream the video. Note that a video can be optimized for different target video qualities as long as it does not exceed the optimal target video quality for the given conditions. Otherwise, it can cause frequently switching to lower qualities and because of already trying to download the segment in desired quality, result in even worse QoE than it has been experienced by DASH.

\section{PERFORMANCE EVALUATION}

QoE-aware streaming was implemented in Matlab simulator, while DASH streaming was evaluated using the WebM DASH player ${ }^{3}$ over the trace-based shaped bandwidth. Bandwidth was shaped using tc command on Linux machine, using a mobile user data rate trace to periodically set the new maximum data rate value. Streaming was executed in Google Chrome browser on the same machine, while video chunks and the streamer resided on the server machine that was in the same LAN as the client. Details about video encodings, access channels, and video quality measurements are described in [3].

Figure 2 shows bandwidth savings and $p_{M A X}$ obtained with QoE-aware streaming of three 4 minute long videos optimized for different target qualities for Samsung Galaxy S3 phone and DASH streaming of these videos encoded in 4 resolutions (720p,480p,360p,240p) over the same data rates. It can be observed that $p_{M A X}$ increases with target perceptual video quality, $M O S^{t}$, depicted as a number above QoE-aware streaming points. Higher bandwidth savings can be achieved for lower $p_{M A X}$, going up to $20 \%$ for $M O S^{\circ}$ in this example.

\footnotetext{
${ }^{2}$ The channel's average data rate can be obtained in several different ways before the streaming starts: (1) from a mobile network operator by committing to support this service, (2) by crowdsourcing of data rates if multiple users on the same location are using this service, or (3) if a user is viewing longer video on YouTube, using the data rates computed from previous seconds to predict the average data rate for the next video playback seconds.

${ }^{3}$ It was chosen for being able to streaming videos in webm format.
}

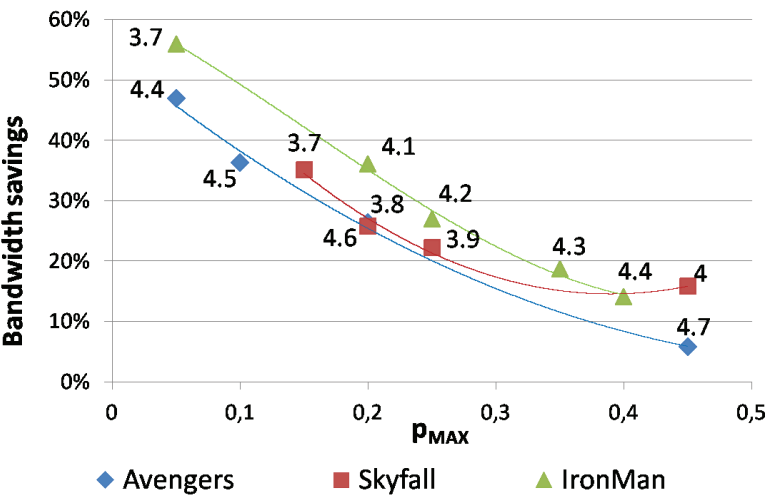

Fig. 2: Bandwidth savings and $p_{M A X}$ across percentiles for videos streamed over channel 1 with different $M O S^{t}$

Figure 3 illustrates a prediction error, err, obtained as a difference in perceptual video quality, evaluated in different percentiles, that can be experienced by choosing the predicted instead of experimentally obtained optimal target video quality, represented by $\widehat{M O S}^{\circ}$ and $M O S^{\circ}$, respectively. The results show that QoE resulting from this prediction differs at most 0.28 MOS points from the maximum achievable QoE.

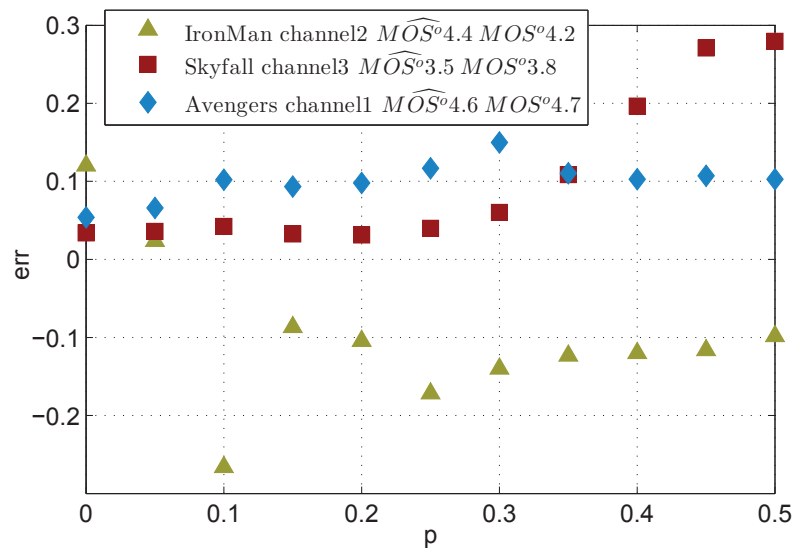

Fig. 3: Prediction error of optimal target MOS across percentiles for three videos streamed over three channels

\section{CONCLUSION}

This paper proposed a streaming method of videos optimized for particular video quality [3] and access channel conditions. The results show that it can improve the worst video qualities caused by DASH and reduce video stream size.

\section{REFERENCES}

[1] N. Cranley, P. Perry, and L. Murphy, "User Perception of Adapting Video Quality," International Journal of Human-Computer Studies, vol. 64, pp. 637-647, 2006

[2] M. Zink, O. Kunzel, J. Schmitt, and R. Steinmetz, "Subjective Impression of Variations in Layer Encoded Videos," in Proc. of IEEE IWQoS, Monterrey, California, June 2003, pp. 137-154.

[3] A. Devlic, P. Kamaraju, P. Lungaro, Z. Segall, and K. Tollmar, "QoEaware optimization for video delivery and storage," in Proceedings of IEEE WOWMOM, Jun. 2015.

[4] J. Yao, S. S. Kanhere, I. Hossain, and M. Hassan, "Empirical Evaluation of HTTP Adaptive Streaming under Vehicular Mobility," In Proc. of IFIP NETWORKING, vol. part 1, pp. 92-105, 2011. 